\title{
ON MEAN VALUE RESULTS FOR THE RIEMANN ZETA-FUNCTION IN SHORT INTERVALS
}

\author{
Aleksandar IVIĆ
}

To R. Balasubramanian and K. Ramachandra, for publishing the HRJ for so many years

Abstract. We discuss the mean values of the Riemann zeta-function $\zeta(s)$, and analyze upper and lower bounds for

$$
\int_{T}^{T+H}\left|\zeta\left(\frac{1}{2}+i t\right)\right|^{2 k} \mathrm{~d} t \quad(k \in \mathbb{N} \text { fixed, } 1 \ll H \leqslant T) .
$$

In particular, the author's new upper bound for the above integral under the Riemann hypothesis is presented.

\section{INTRODUCTION}

The aim of this paper is to analyze bounds for the $2 k$-th moment of the Riemann zeta-function $\zeta(s)$ in "short" intervals. Recall that (henceforth $s=\sigma+i t$ will denote a complex variable)

$$
\zeta(s)=\sum_{n=1}^{\infty} n^{-s} \quad(\sigma>1),
$$

and for other values of $s$ the zeta-function is defined by analytic continuation. For all values of $s \in \mathbb{C}$ we have the functional equation

$$
\zeta(s)=\chi(s) \zeta(1-s), \quad \chi(s)=2^{s} \pi^{s-1} \sin \left(\frac{1}{2} \pi s\right) \Gamma(1-s),
$$

where

$$
\chi(s)=\left(\frac{2 \pi}{t}\right)^{\sigma+i t-1 / 2} \mathrm{e}^{i(t+\pi / 4)}\left(1+O\left(\frac{1}{t}\right)\right) \quad\left(t \geqslant t_{0}>0\right) .
$$

Our main object of study is $I_{\sigma}(T+H, k)-I_{\sigma}(T, k)$, where

$$
I_{\sigma}(T, k):=\int_{1}^{T}|\zeta(\sigma+i t)|^{2 k} \mathrm{~d} t \quad(\sigma \in \mathbb{R}, k \in \mathbb{N} \text { fixed }) .
$$

Actually in (1.3) one can consider any $k>0$, but the integral is considerably less difficult to deal with when $k \in \mathbb{N}$, since $|z|^{2}=z \cdot \bar{z}$. In view of the functional equation (1.1) it suffices to consider the evaluation of $I_{\sigma}(T+H, k)-I_{\sigma}(T, k)$ in the following four cases:

1991 Mathematics Subject Classification. 11M06.

Key words and phrases. Riemann zeta-function, mean values, short intervals. 
1. $\sigma>1$.

2. $\sigma=1$.

3. The case of the "critical strip" $\frac{1}{2}<\sigma<1$.

4. The case of the "critical line" $\sigma=\frac{1}{2}$.

The last case is by far the most important one. The hitherto unproved Riemann Hypothesis, probably the most famous open problem in all Mathematics (henceforth $\mathrm{RH}$ for short), states that all complex zeros of $\zeta(s)$ have real parts equal to $\frac{1}{2}$. The zeros $s=-2,-4, \ldots$, which come from the sine-factor in the functional equation in (1.1), are usually called the "trivial zeros" of $\zeta(s)$.

The aim of this paper is to present results on $I_{\sigma}(T+H, k)-I_{\sigma}(T, k)$. We signal that there are two monographs dedicated solely to the mean values of $\zeta(s)$, namely the author's [12] and K. Ramachandra's [31]. Coupled with the standard monographs [11] and [34], which provide many additional references and historical background, they provide the basic literature on the subject. To avoid excessive length of the text, many important subjects and references had to be omitted. The accent is on the lower bounds when $\frac{1}{2} \leqslant \sigma<1$, which are unconditional, and the new upper bound which can be only attained if the $\mathrm{RH}$ is assumed. Besides this, asymptotic formulas for the mean square and fourth power have been presented in somewhat more detail.

\section{THE CASE WHEN $\sigma>1$}

We start with the case when $\sigma>1$. This is actually the only easy case, and thus the discussion can be given in detail. We have

$$
\zeta^{k}(s)=\sum_{n=1}^{\infty} d_{k}(n) n^{-s} \quad(\sigma>1),
$$

where the divisor function $d_{k}(n)$ denotes the number of ways $n$ may be represented as a product of $k$ factors, so that in particular

$$
d_{2}(n) \equiv d(n)=\sum_{\delta \mid n} 1
$$

is the number of positive divisors of $n$. For fixed $k \in \mathbb{N}$ and fixed $\sigma>1$ we have

$$
\begin{aligned}
& \int_{1}^{T}|\zeta(\sigma+i t)|^{2 k} \mathrm{~d} t=\int_{1}^{T} \zeta^{k}(\sigma+i t) \zeta^{k}(\sigma-i t) \mathrm{d} t \\
& =\int_{1}^{T} \sum_{m=1}^{\infty} \sum_{n=1}^{\infty} d_{k}(m) d_{k}(n)(m n)^{-\sigma}\left(\frac{m}{n}\right)^{i t} \mathrm{~d} t .
\end{aligned}
$$

We group the terms $m=n$ (the so-called "diagonal terms') together and the terms $m \neq n$ (the "non-diagonal" terms), noting that $d_{k}(n) \ll_{\varepsilon, k} n^{\varepsilon}$. The former trivially produce

$$
(T-1) \sum_{n=1}^{\infty} d_{k}^{2}(n) n^{-2 \sigma}
$$


The latter ones are integrated and make a contribution which is

$$
\begin{aligned}
& \ll \sum_{m=1}^{\infty} \sum_{n=1, n<m}^{\infty} d_{k}(m) d_{k}(n)(m n)^{-\sigma}\left(\log \left(\frac{m}{n}\right)\right)^{-1} \\
& \ll \sum_{n=1}^{\infty} d_{k}(n) n^{-\sigma}\left(\sum_{n<m<2 n} d_{k}(m) m^{-\sigma}\left(\log \left(\frac{m}{n}\right)\right)^{-1}+1\right) \\
& \ll \sum_{n=1}^{\infty} n^{2 \varepsilon-\sigma} \sum_{n<m<2 n} \frac{m^{1-\sigma}}{m-n}+1 \ll 1,
\end{aligned}
$$

if $\varepsilon$ is small enough. This finally gives the asymptotic formula

$$
\int_{1}^{T}|\zeta(\sigma+i t)|^{2 k} \mathrm{~d} t=T C(k, \sigma)+O(1), \quad C(k, \sigma):=\sum_{n=1}^{\infty} d_{k}^{2}(n) n^{-2 \sigma} .
$$

Hence from (1.3) and (2.1) we have

$$
I_{\sigma}(T+H, k)-I_{\sigma}(T, k)=C(k, \sigma) H+O(1) \quad(1 \ll H \leqslant T) .
$$

\section{THE CASE WHEN $\sigma=1$}

In the case when $\sigma=1, k=1$ we have precise results due to R. Balasubramanian, A. Ivić and K. Ramachandra [3]. Namely, let

$$
R(T):=\int_{1}^{T}|\zeta(1+i t)|^{2} \mathrm{~d} t-\zeta(2) T+\pi \log T .
$$

The lower limit of integration, similarly as in (2.1), is taken to be unity (although any positive number would clearly do as well), since $s=1$ is the pole of $\zeta(s)$.

The function $R(T)$ in (3.1) may be considered as an error term, since it was shown (op. cit.) that

$$
\begin{aligned}
R(T) & =O\left((\log T)^{2 / 3}(\log \log T)^{1 / 3}\right), \\
\int_{1}^{T} R(t) \mathrm{d} t & =O(T), \\
\int_{1}^{T} R^{2}(t) \mathrm{d} t & =O\left(T(\log \log T)^{4}\right) .
\end{aligned}
$$

The exponent $2 / 3$ for the pointwise bound for $R(T)$ in $(3.2)$ is connected to the best known bound for $\zeta(1+i t)$ (see e.g., [11, Chapter 6$]$ ), namely

$$
\zeta(1+i t) \ll(\log t)^{2 / 3} \quad(t>3) .
$$

In [4] R. Balasubramanian, A. Ivić and K. Ramachandra show that, if $k$ is any complex constant, then ( $p$ denotes primes)

$$
\begin{aligned}
& \int_{1}^{T}\left|\zeta^{k}(1+i t)\right|^{2} \mathrm{~d} t=T \sum_{n=1}^{\infty}\left|d_{k}(n)\right|^{2} n^{-2}+O\left((\log T)^{|k|^{2}}\right), \\
& \int_{1}^{T}\left|\frac{\zeta^{\prime}(1+i t)}{\zeta(1+i t)}\right|^{2} \mathrm{~d} t=T \sum_{m=1}^{\infty} \sum_{p} \frac{\log ^{2} p}{p^{2 m}}+O\left((\log T)^{2}\right), \\
& \int_{1}^{T}|\log \zeta(1+i t)|^{2} \mathrm{~d} t=T \sum_{m=1}^{\infty} \sum_{p}\left(m p^{m}\right)^{-2}+O(\log \log T) .
\end{aligned}
$$


While the proof of (3.2) was essentially elementary, the proof of (3.3) used complex integration over the so called Hooley-Huxley contour, as modified by K. Ramachandra [30]. From (3.3) we have the asymptotic formula $(1 \ll H \leqslant T)$

$$
\left.I_{1}(T+H, k)-I_{1}(T, k)=c(k) H+O\left((\log T)^{|k|^{2}}\right)\right), \quad c(k):=\sum_{n=1}^{\infty}\left|d_{k}(n)\right|^{2} n^{-2} .
$$

4. The MOMENTS IN THE CRITICAL STRIP $\frac{1}{2}<\sigma<1$

As $\sigma$ moves further to the left from $\sigma=1$ to the critical line $\sigma=\frac{1}{2}$, the results on $I_{\sigma}(T, k)$ become richer. Mean values of $\zeta(s)$ for $\frac{1}{2} \leqslant \sigma<1$ in the cases $k=1$ and $k=2$ have been extensively studied, and represent one of the central themes in zeta-function theory. For the mean square case the reader should consult the survey paper of K. Matsumoto [26]. One has (see Ivić-Matsumoto [20])

$$
\begin{aligned}
\int_{1}^{T}|\zeta(\sigma+i t)|^{2} \mathrm{~d} t & =\zeta(2 \sigma) T+\frac{\zeta(2 \sigma-1) \Gamma(2 \sigma-1)}{1-\sigma} \sin (\pi \sigma) T^{2-2 \sigma} \\
& +E_{\sigma}(T) \quad\left(\frac{1}{2}<\sigma \leqslant 1\right),
\end{aligned}
$$

where the error-term function $E_{\sigma}(T)$ satisfies the bound

$$
E_{\sigma}(T)=O\left(T^{2(1-\sigma) / 3} \log ^{2 / 9} T\right) \quad\left(\frac{1}{2}<\sigma \leqslant 1\right)
$$

Several other results (op. cit.) involving $E_{\sigma}(T)$ are known. They include mean square and omega results, for which the reader should also consult K. Matsumoto [25] and Matsumoto-Meurman [27].

For the fourth moment we have (see [14, Theorem 2])

$$
\int_{1}^{T}|\zeta(\sigma+i t)|^{4} \mathrm{~d} t=\frac{\zeta^{2}(2 \sigma)}{\zeta(4 \sigma)} T+O\left(T^{2-2 \sigma} \log ^{3} T\right) \quad\left(\frac{1}{2}<\sigma \leqslant 1\right)
$$

which is the sharpest hitherto published asymptotic formula valid in the whole range $\frac{1}{2}<\sigma \leqslant 1$. In [23] Y. Motohashi and the author used methods from spectral theory to obtain several results on $I_{\sigma}(T, 2)$. They proved that, if $\sigma$ is a fixed number such that $\frac{1}{2}<\sigma<\frac{3}{4}$, then with suitable constants $a_{j}(\sigma)\left(a_{2}(\sigma)>0\right)$ we have

$$
\begin{aligned}
(4.3) \int_{1}^{T}|\zeta(\sigma+i t)|^{4} \mathrm{~d} t & =\frac{\zeta^{4}(2 \sigma)}{\zeta(4 \sigma)} T+\frac{T}{3-4 \sigma}\left(\frac{T}{2 \pi}\right)^{2-4 \sigma} \frac{\zeta^{4}(2-2 \sigma)}{\zeta(4-4 \sigma)} \\
& +T^{2-2 \sigma}\left(a_{0}(\sigma)+a_{1}(\sigma) \log T+a_{2}(\sigma) \log ^{2} T\right)+E_{2}(T, \sigma)
\end{aligned}
$$

where, with some $C>0$, the error-term function $E_{2}(T, \sigma)$ satisfies

$$
E_{2}(T, \sigma) \ll T^{2 /(1+4 \sigma)} \log ^{C} T \quad\left(\frac{1}{2}<\sigma<\frac{3}{4}\right) .
$$

Note that $3-4 \sigma>2 /(1+4 \sigma)$ for $\frac{1}{2}<\sigma<\frac{1+\sqrt{2}}{4}$ and that $2-2 \sigma>2 /(1+4 \sigma)$ for $\sigma<\frac{3}{4}$. Thus the bound for the error term $E_{2}(T, \sigma)$ in (4.4) is already larger than the second main term in (4.3) unless $\frac{1}{2}<\sigma<\frac{1+\sqrt{2}}{4}$. 
All of the above results were been obtained by special methods, and cannot be generalized to higher moments. The formula for the general $2 k$-th moment of $\zeta(s)$ can be conveniently written (cf. [11, Chapter 8]) as

$$
\int_{1}^{T}|\zeta(\sigma+i t)|^{2 k} \mathrm{~d} t=\sum_{n=1}^{\infty} d_{k}^{2}(n) n^{-2 \sigma} T+R(k, \sigma ; T), \quad R(k, \sigma ; T)=o(T)
$$

where $\frac{1}{2}<\sigma_{0}(k) \leqslant \sigma \leqslant 1, T \rightarrow \infty$. It was proved (op. cit.) that

$$
R(k, \sigma ; T) \ll_{\varepsilon} T^{\frac{2-\sigma-\sigma_{k}^{*}}{2-2 \sigma_{k}^{*}}+\varepsilon} \quad\left(\sigma_{k}^{*}<\sigma<1\right)
$$

where henceforth $\varepsilon$ denotes arbitrarily small constants, not necessarily the same ones at each occurrence, and $\sigma_{k}^{*}$ is the infimum of $\sigma^{*}\left(\geqslant \frac{1}{2}\right)$ for which

$$
\int_{1}^{T}\left|\zeta\left(\sigma^{*}+i t\right)\right|^{2 k} \mathrm{~d} t \ll_{\varepsilon} T^{1+\varepsilon}
$$

holds for any given $\varepsilon$. Writing further the bounds for $R(k, \sigma ; T)$ in the form

$$
R(k, \sigma ; T) \ll_{\varepsilon} T^{c_{k}(\sigma)+\varepsilon}
$$

and using the known bounds for $\sigma_{k}^{*}$ when $3 \leqslant k \leqslant 6$, it follows from (4.6) that we have

$$
\begin{array}{llll}
c_{3}(\sigma)=\frac{17-12 \sigma}{10} & \left(\frac{7}{12}<\sigma<1\right), & c_{4}(\sigma)=\frac{11-8 \sigma}{6} & \left(\frac{5}{8}<\sigma<1\right), \\
c_{5}(\sigma)=\frac{79-60 \sigma}{38} & \left(\frac{41}{60}<\sigma<1\right), & c_{6}(\sigma)=\frac{9-7 \sigma}{4} & \left(\frac{5}{7}<\sigma<1\right) .
\end{array}
$$

As indicated in [11], explicit values for $c_{k}(\sigma)$ could be given for any fixed $k>1$, but the expressions in general would be cumbersome, so only explicit values were given for $2 \leqslant k \leqslant 6$. The point of (4.5)-(4.7) lies in the fact that each value of $c_{k}(\sigma)$ satisfies $c_{k}(\sigma)<1$ (i.e., when (4.5) becomes a true asymptotic formula), precisely for the range given in (4.7). However, as $\sigma$ approaches 1 , the values of $c_{k}(\sigma)$ become rather poor and they do not tend to zero, as one expects.

New bounds for $R(k, \sigma ; T)$ have been given by the author in [18]. To formulate them let, as usual,

$$
\beta_{k}=\inf \left\{b_{k}(\geqslant 0): \int_{1}^{x} \Delta_{k}^{2}(y) \mathrm{d} y \ll x^{1+2 b_{k}}\right\},
$$

where

$$
\Delta_{k}(x)=\sum_{n \leqslant x} d_{k}(n)-x P_{k-1}(\log x)
$$

is the error term in the asymptotic formula for the summatory function of $d_{k}(n)$ (see e.g., [11, Chapter 13] for a comprehensive account). Here $P_{k-1}(y)$ is a polynomial of degree $k-1$, whose coefficients (which depend on $k$ ) may be explicitly evaluated. 
Then, for fixed $\sigma$ satisfying $\max \left(\beta_{k}, \frac{1}{2}\right)<\sigma<1$ and every fixed integer $k \geqslant 3$, we have

$$
R(k, \sigma ; T) \ll_{\varepsilon} T^{\frac{2(1-\sigma)}{1-\beta_{k}}+\varepsilon},
$$

while for fixed $\sigma$ satisfying $\sigma_{k}^{*}<\sigma<1$ and every fixed integer $k \geqslant 3$, we have

$$
R(k, \sigma ; T) \ll_{\varepsilon} T^{\frac{2(1-\sigma)}{2-\sigma_{k}^{*}-\sigma}+\varepsilon} .
$$

Note that (4.9) will provide good results for values of $\sigma$ close to 1 . On the other hand, (4.10) gives good bound for $R(k, \sigma ; T)$ when $\sigma$ is close to $\sigma_{k}^{*}$. From the above results it follows that we have

$$
\begin{array}{ll}
\int_{1}^{T}|\zeta(\sigma+i t)|^{6} \mathrm{~d} t=T \sum_{n=1}^{\infty} d_{3}^{2}(n) n^{-2 \sigma}+O_{\varepsilon}\left(T^{3(1-\sigma)+\varepsilon}\right) & \left(\frac{1}{2}<\sigma<1\right), \\
\int_{1}^{T}|\zeta(\sigma+i t)|^{8} \mathrm{~d} t=T \sum_{n=1}^{\infty} d_{4}^{2}(n) n^{-2 \sigma}+O_{\varepsilon}\left(T^{\frac{16}{5}(1-\sigma)+\varepsilon}\right) & \left(\frac{1}{2}<\sigma<1\right), \\
\int_{1}^{T}|\zeta(\sigma+i t)|^{10} \mathrm{~d} t=T \sum_{n=1}^{\infty} d_{5}^{2}(n) n^{-2 \sigma}+O_{\varepsilon}\left(T^{\frac{40}{11}(1-\sigma)+\varepsilon}\right) & \left(\frac{1}{2}<\sigma<1\right), \\
\int_{1}^{T}|\zeta(\sigma+i t)|^{12} \mathrm{~d} t=T \sum_{n=1}^{\infty} d_{6}^{2}(n) n^{-2 \sigma}+O_{\varepsilon}\left(T^{4(1-\sigma)+\varepsilon}\right) & \left(\frac{1}{2}<\sigma<1\right),
\end{array}
$$

while if the Lindelöf hypothesis (that $\left.\zeta\left(\frac{1}{2}+i t\right) \ll_{\varepsilon} t^{\varepsilon}\right)$ is true then, for $\sigma>\frac{1}{2}$, (4.11)

$$
\int_{1}^{T}|\zeta(\sigma+i t)|^{2 k} \mathrm{~d} t=\sum_{n=1}^{\infty} d_{k}^{2}(n) n^{-2 \sigma} T+O_{\varepsilon}\left(T^{\frac{4 k(1-\sigma)}{k+1}+\varepsilon}\right)\left(\frac{1}{2}<\sigma \leqslant 1, k \geqslant 2\right) .
$$

In fact, the bound (4.11) for all $k \in \mathbb{N}$ is equivalent to the Lindelöf hypothesis. One can easily obtain from the above results asymptotic formulas for $I_{\sigma}(T+H, k)-$ $I_{\sigma}(T, k)$ for certain range of $H=H(\sigma, k)$. However, as far as lower bounds are concerned, one can do considerably better.

\section{THE LOWER BOUND FOR $\frac{1}{2}<\sigma<1$}

We have the following

THEOREM 1. If $k \geqslant 1$ is a fixed integer, $\sigma \geqslant \frac{1}{2}$ is fixed, $12 \log \log T \leqslant H \leqslant$ $T, T \geqslant T_{0}>0$, then uniformly in $\sigma$

$$
\int_{T-H}^{T+H}|\zeta(\sigma+i t)|^{k} \mathrm{~d} t \gg H
$$

Proof. This result is due to Balasubramanian-Ramachandra (see [31], and also [12, Chapter 1] for a proof). Let $\sigma_{1}=\sigma+2, s_{1}=\sigma_{1}+i t, T-\frac{1}{2} H \leqslant t \leqslant T+\frac{1}{2} H$. Then $\zeta\left(s_{1}\right) \gg 1$ and therefore

$$
\int_{T-\frac{1}{2} H}^{T+\frac{1}{2} H}\left|\zeta\left(\sigma_{1}+i t\right)\right|^{k} \mathrm{~d} t \gg H
$$


Let now $\mathcal{E}$ be the rectangle with vertices $\sigma+i T \pm i H, \sigma_{2}+i T \pm i H\left(\sigma_{2}=\sigma+3\right)$ and let $X$ be a parameter which satisfies

$$
T^{-c} \leqslant X \leqslant T^{c}
$$

for some constant $c>0$. The residue theorem gives then

$$
\frac{1}{\mathrm{e}} \zeta^{k}\left(s_{1}\right)=\frac{1}{2 \pi i} \int_{\mathcal{E}} \frac{\zeta^{k}(w)}{w-s_{1}} \exp \left(-\cos \left(\frac{w-s_{1}}{3}\right)\right) X^{s_{1}-w} \mathrm{~d} w
$$

On $\mathcal{E}$ we have $\mid \Re$ e $\frac{1}{3}\left(w-s_{1}\right) \mid \leqslant 1$, and on its horizontal sides

$$
\left|\Im \mathrm{m}\left(\frac{w-s_{1}}{3}\right)\right| \geqslant \frac{1}{3} \cdot \frac{H}{2} \geqslant 2 \log \log T
$$

Note that for $w=u+i v(u, v \in \mathbb{R})$ we have

$$
\begin{aligned}
|\exp (-\cos w)| & =\left|\exp \left(-\frac{1}{2}\left(\mathrm{e}^{i w}+\mathrm{e}^{-i w}\right)\right)\right| \\
& =\left|\exp \left(-\frac{1}{2}\left(\mathrm{e}^{i u} \mathrm{e}^{-v}+\mathrm{e}^{-i u} \mathrm{e}^{v}\right)\right)\right|=\exp (-\cos u \cdot \cosh v) .
\end{aligned}
$$

The above function, which may be justly called Ramachandra's kernel function, sets the limit to the lower bound for $H$ (a multiple of $\log \log T$ ) in Theorem 1. Actually, according to W.K. Hayman, there does not exist a regular function of a similar type which decreases faster than a double-order exponential. Therefore Ramachandra's kernel function is optimal. This does not preclude that the lower bound in Theorem 1 for $H$ holds for some function smaller than a multiple of $\log \log T$, but such a result (if true) certainly cannot be obtained be a variant of the above method. It is an interesting problem to determine the optimal lower bound for $H$ in Theorem 1 and Theorem 2 (see Section 7) for which the assertions in question hold true.

To continue with the proof of the theorem, note then that, if $w$ lies on the horizontal sides of $\mathcal{E}$, we have

$$
\begin{aligned}
& \left|\exp \left(-\cos \left(\frac{w-s_{1}}{3}\right)\right)\right| \\
& \leqslant \cos \left(-\frac{1}{2} \cos 1 \exp (2 \log \log T)\right)=\exp \left(-\frac{1}{2} \cos 1(\log T)^{2}\right) .
\end{aligned}
$$

Therefore the condition $T^{-c} \leqslant X \leqslant T^{c}$ ensures that, for suitable $C, c_{1}>0$,

$$
\begin{aligned}
\zeta^{k}\left(\sigma_{1}+i t\right) & \ll X^{2} \int_{T-H}^{T+H}|\zeta(\sigma+i v)|^{k} \exp \left(-c_{1} \mathrm{e}^{|v-t| / 3}\right) \mathrm{d} v \\
& +X^{-1} \int_{T-H}^{T+H} \exp \left(-c_{1} \mathrm{e}^{|v-t| / 3}\right) \mathrm{d} v+\mathrm{e}^{-C \log ^{2} T}
\end{aligned}
$$


Integrating this estimate over $t \in\left[T-\frac{1}{2} H, T+\frac{1}{2} H\right]$ and using (5.2) we obtain

$$
\begin{aligned}
H & \ll X^{2} \int_{T-H}^{T+H}|\zeta(\sigma+i v)|^{k} \mathrm{~d} v\left(\int_{T-\frac{1}{2} H}^{T+\frac{1}{2} H} \exp \left(-c_{1} \mathrm{e}^{|v-t| / 3}\right) \mathrm{d} t\right) \\
& +X^{-1} \int_{T-H}^{T+H} \mathrm{~d} v\left(\int_{T-\frac{1}{2} H}^{T+\frac{1}{2} H} \exp \left(-c_{1} \mathrm{e}^{|v-t| / 3}\right) \mathrm{d} t\right) \\
& \ll X^{2} \int_{T-H}^{T+H}|\zeta(\sigma+i v)|^{k} \mathrm{~d} v+X^{-1} H .
\end{aligned}
$$

Let now

$$
I:=\int_{T-H}^{T+H}|\zeta(\sigma+i v)|^{k} \mathrm{~d} v
$$

and choose $X=H^{\varepsilon}$. Then (5.4) gives $I \gg H^{1-2 \varepsilon}$, showing that $I$ cannot be too small. Then we choose $X=H^{1 / 3} I^{-1 / 3}$, so that (since $\zeta\left(\frac{1}{2}+i t\right) \ll|t|^{1 / 6}$ ) trivially

$$
T^{-k / 18} \ll X \ll H \ll T,
$$

and (5.3) is satisfied. With this choice of $X$ (5.4) reduces to $H \ll H^{2 / 3} I^{1 / 3}$, and (5.1) follows.

\section{The MeAn VAlues FOR $\sigma=\frac{1}{2}$}

Asymptotic formulas for $I_{1 / 2}(T, k)$ are known to exist only in the cases when $k=1$ or $k=2$. For the mean square it is customary to define

$$
E(T)=\int_{0}^{T}\left|\zeta\left(\frac{1}{2}+i t\right)\right|^{2} \mathrm{~d} t-T\left(\log \left(\frac{T}{2 \pi}\right)+2 \gamma-1\right),
$$

where $\gamma=-\Gamma^{\prime}(1)=0.5772156649 \ldots$ is Euler's constant. The fundamental result on this subject is the work of F.V. Atkinson [1], who established an explicit formula for $E(T)$. Atkinson's result is the following: let $0<A<A^{\prime}$ be any two fixed constants such that $A T<N<A^{\prime} T$, and let $N^{\prime}=N^{\prime}(T)=T /(2 \pi)+N / 2-$ $\left(N^{2} / 4+N T /(2 \pi)\right)^{1 / 2}$. Then

$$
E(T)=\Sigma_{1}(T)+\Sigma_{2}(T)+O\left(\log ^{2} T\right)
$$

where

$$
\Sigma_{1}(T)=2^{1 / 2}\left(\frac{T}{2 \pi}\right)^{1 / 4} \sum_{n \leqslant N}(-1)^{n} d(n) n^{-3 / 4} e(T, n) \cos (f(T, n))
$$

$$
\Sigma_{2}(T)=-2 \sum_{n \leqslant N^{\prime}} d(n) n^{-1 / 2}\left(\log \frac{T}{2 \pi n}\right)^{-1} \cos \left(T \log \left(\frac{T}{2 \pi n}\right)-T+\frac{1}{4} \pi\right)
$$


with

$$
\begin{aligned}
& f(T, n)=2 T \operatorname{arsinh}(\sqrt{\pi n /(2 T)})+\sqrt{2 \pi n T+\pi^{2} n^{2}}-\frac{1}{4} \pi \\
& =-\frac{1}{4} \pi+2 \sqrt{2 \pi n T}+\frac{1}{6} \sqrt{2 \pi^{3}} n^{3 / 2} T^{-1 / 2}+a_{5} n^{5 / 2} T^{-3 / 2}+a_{7} n^{7 / 2} T^{-5 / 2}+\ldots,
\end{aligned}
$$

$$
\begin{aligned}
e(T, n) & =(1+\pi n /(2 T))^{-1 / 4}\left\{(2 T / \pi n)^{1 / 2} \operatorname{arsinh}(\sqrt{\pi n /(2 T)})\right\}^{-1} \\
& =1+O(n / T) \quad(1 \leqslant n<T)
\end{aligned}
$$

and $\operatorname{ar} \sinh x=\log \left(x+\sqrt{1+x^{2}}\right)$. Another explicit formula for $E(T)$, due to $\mathrm{R}$. Balasubramanian [2], is based on the use of the classical Riemann-Siegel formula for $\zeta\left(\frac{1}{2}+i t\right)$ (see $[11$, eq. (4.5)] or [34, Theorem 4.16]). This is

$$
\begin{aligned}
E(T) & =2 \sum_{n \leqslant K} \sum_{m \leqslant K, m \neq n} \frac{\sin (T \log n / m)}{\sqrt{m n} \log n / m} \\
& +2 \sum_{n \leqslant K} \sum_{m \leqslant K, m \neq n} \frac{\sin \left(2 \theta_{1}-T \log m n\right)}{\sqrt{m n}\left(2 \theta_{1}^{\prime}-\log m n\right)}+O\left(\log ^{2} T\right),
\end{aligned}
$$

where $\theta_{1}=\theta_{1}(T)=\frac{1}{2} T \log (T /(2 \pi))-\frac{1}{2} T-\frac{1}{8} \pi, K=\sqrt{T /(2 \pi)}$. Although structurally different, both (6.2)-(6.6) and (6.7) can be used to estimate $E(T)$ and, indirectly $\left|\zeta\left(\frac{1}{2}+i t\right)\right|$, in view of the bound (see e.g., [12, Chapter 1])

$$
\left|\zeta\left(\frac{1}{2}+i t\right)\right|^{2} \ll \log t\left(\int_{t-1}^{t+1}\left|\zeta\left(\frac{1}{2}+i x\right)\right|^{2} \mathrm{~d} x+1\right) \ll \log t(\log t+E(t+1)-E(t-1)) .
$$

The sharpest known bounds for $\zeta\left(\frac{1}{2}+i t\right)$ and $E(T)$ are discussed in the paper of Huxley-Ivić [9], where the new result of N.G. Watt that

$$
E(T) \ll_{\varepsilon} T^{131 / 416+\varepsilon} \quad\left(\frac{131}{416}=0.314903 \ldots\right)
$$

is announced. Thus unconditionally we have that

$$
I_{1 / 2}(T+H, 1)-I_{1 / 2}(T-H, 1)=\int_{T-H}^{T+H}\left|\zeta\left(\frac{1}{2}+i t\right)\right|^{2} \mathrm{~d} t=(2 \log T+O(1)) H
$$

for $H=T^{\theta}, \theta>131 / 416$. For a wealth of other results concerning $E(T)$ the reader should consult [11],[12]. In particular (see Hafner-Ivić [4] and [12, Chapter 3]), we have

$$
E(T)=\Omega_{+}\left\{(T \log T)^{1 / 4}(\log \log T)^{(3+\log 4) / 4)} \mathrm{e}^{-C \sqrt{\log \log \log T}}\right\}
$$

for some $C>0$ and, for some $D>0$,

$$
E(T)=\Omega_{-}\left\{T^{1 / 4} \exp \left(\frac{D(\log \log T)^{1 / 4}}{(\log \log \log T)^{3 / 4}}\right)\right\} .
$$


Here, as usual, $f=\Omega_{+}(g)$ means $\lim \sup f / g>0$, and $f=\Omega_{-}(g)$ means that $\liminf f / g<0$ holds. In the other direction, it is commonly conjectured that $E(T) \ll_{\varepsilon} T^{1 / 4+\varepsilon}$ holds.

The case of the asymptotic formula for $I_{1 / 2}(T, k)$ when $k=2$ is more involved. This formula is customarily written as

$$
\int_{0}^{T}\left|\zeta\left(\frac{1}{2}+i t\right)\right|^{4} \mathrm{~d} t=T p_{4}(\log T)+E_{2}(T), \quad p_{4}(x)=\sum_{j=0}^{4} a_{j} x^{j}
$$

A classical result of A.E. Ingham [10] from 1926 is that $a_{4}=1 /\left(2 \pi^{2}\right)$ and that the error term $E_{2}(T)$ satisfies the bound $E_{2}(T) \ll T \log ^{3} T$. In 1979 D.R. Heath-Brown [8] improved this to $E_{2}(T) \ll_{\varepsilon} T^{\frac{7}{8}+\varepsilon}$. He also calculated

$$
a_{3}=2\left(4 \gamma-1-\log (2 \pi)-12 \zeta^{\prime}(2) \pi^{-2}\right) \pi^{-2}
$$

and produced more complicated expressions for $a_{0}, a_{1}$ and $a_{2}$. The author [13] made an explicit evaluation of $a_{0}, a_{1}, a_{2}$. Modern approaches to the study of $E_{2}(T)$ involve the use of spectral theory of the non-Euclidean Laplacian. For a comprehensive account on this topic and its applications to $\zeta(s)$ the reader is referred to Y. Motohashi's monograph [29]. The fundamental result is Y. Motohashi's explicit formula [28] for

$$
I(T, \Delta):=\frac{1}{\Delta \sqrt{\pi}} \int_{-\infty}^{\infty}\left|\zeta\left(\frac{1}{2}+i T+i t\right)\right|^{4} \mathrm{e}^{-t^{2} \Delta^{-2}} \mathrm{~d} t
$$

under the condition

$$
0<\Delta \leqslant \frac{T}{\log T}
$$

The presence of the Gaussian smoothing factor $\mathrm{e}^{-x^{2}}$ enabled Motohashi to deal with various convergence problems occurring in the proof. In simplified form, the formula reads

$$
\begin{aligned}
I(T, \Delta) & =\pi 2^{-1 / 2} T^{-1 / 2} \sum_{j=1}^{\infty} \alpha_{j} \kappa_{j}^{-1 / 2} H_{j}^{3}\left(\frac{1}{2}\right) \sin \left(\kappa_{j} \log \left(\frac{\kappa_{j}}{4 \mathrm{e} T}\right)\right) \mathrm{e}^{-\left(\Delta \kappa_{j} / 2 T\right)^{2}} \\
& +O\left(\log ^{C} T\right) .
\end{aligned}
$$

Here the discrete spectrum of the non-Euclidean Laplace operator, acting on the full modular group, has the form $\left\{\lambda_{j}\right\}_{j=1}^{\infty}, \lambda_{j}=\kappa_{j}^{2}+\frac{1}{4}\left(\kappa_{j}>0\right), \rho_{j}(1)$ is the first Fourier coefficient of the Maass wave form attached to $\kappa_{j}$, and

$$
\alpha_{j}=\frac{\left|\rho_{j}(1)\right|^{2}}{\cosh \left(\pi \kappa_{j}\right)}
$$

Further, for arbitrary fixed $A>0, C=C(A)>0$, the above formula holds if $\Delta$ satisfies

$$
\frac{\sqrt{T}}{\log ^{A} T} \leqslant \Delta \leqslant T \exp (-\sqrt{\log T})
$$


The proof of the formula for $I(T, \Delta)$ depends heavily on the use of spectral theory, especially the so-called Bruggeman-Kuznetsov trace formulas, which relate sums of Kloosterman sums to certain sums involving quantities from spectral theory. Y. Motohashi and the author [21]-[22] obtained several results on $E_{2}(T)$ and some related problems (see also the joint paper with M. Jutila [24]). It is known now that $(f=\Omega(g)$ means that $\lim \sup |f| / g>0)$

$$
\begin{gathered}
E_{2}(T)=O\left(T^{2 / 3} \log ^{C_{1}} T\right), \quad E_{2}(T)=\Omega\left(T^{1 / 2}\right), \\
\int_{0}^{T} E_{2}(t) \mathrm{d} t=O\left(T^{3 / 2}\right), \int_{0}^{T} E_{2}^{2}(t) \mathrm{d} t=O\left(T^{2} \log ^{C_{2}} T\right),
\end{gathered}
$$

with effective constants $C_{1}, C_{2}>0$ (the values $C_{1}=8, C_{2}=22$ are admissible). Y. Motohashi [29] improved the omega-result to

$$
E_{2}(T)=\Omega_{ \pm}\left(T^{1 / 2}\right) .
$$

In the other direction, it is commonly conjectured that $E_{2}(T) \ll_{\varepsilon} T^{1 / 2+\varepsilon}$ holds. Thus unconditionally we have that

$$
I_{1 / 2}(T+H, 2)-I_{1 / 2}(T-H, 2)=\int_{T-H}^{T+H}\left|\zeta\left(\frac{1}{2}+i t\right)\right|^{4} \mathrm{~d} t=\frac{H}{\pi^{2}}\left(\log ^{4} T+O\left(\log ^{3} T\right)\right)
$$

for $H=T^{2 / 3} \log ^{C} T$ and some suitable $C>0$.

In what concerns higher moments (i.e., $I_{1 / 2}(T, k)$ for $k \in \mathbb{N}, k>2$ ) there are not many results. The bound of D.R. Heath-Brown [7] that

$$
I_{1 / 2}(T, 6)=\int_{1}^{T}\left|\zeta\left(\frac{1}{2}+i t\right)\right|^{12} \mathrm{~d} t \ll T^{2}(\log T)^{17}
$$

still represents essentially the strongest known result on high moments of $\left|\zeta\left(\frac{1}{2}+i t\right)\right|$. Although with the use of methods relating to random matrix theory (see J.B. Conrey et al. [5]) it is possible to make plausible conjectures for the asymptotic formulas for $I_{1 / 2}(T, k)$ when $k \in \mathbb{N}$ is fixed, no one has proved yet such an asymptotic formula for $k \geqslant 3$, even assuming the Riemann Hypothesis. The Lindelöf Hypothesis is equivalent to the bound

$$
\int_{0}^{T}\left|\zeta\left(\frac{1}{2}+i t\right)\right|^{2 k} \mathrm{~d} t \ll_{k, \varepsilon} T^{1+\varepsilon}
$$

for every $k \geqslant 1$ and any $\varepsilon>0$. There are some other conditional results on $I_{1 / 2}(T, k)$. For example, the author $[15]$ proved that the expected bound

$$
I_{1 / 2}(T, 3)=\int_{0}^{T}\left|\zeta\left(\frac{1}{2}+i t\right)\right|^{6} \mathrm{~d} t \ll_{\varepsilon} T^{1+\varepsilon}
$$

holds if a certain conjecture involving the ternary additive divisor problem (the summatory function of $d_{3}(n) d_{3}(n+h)$ when $h$ is not fixed) holds. 
7. The BOUndS OVER SHORT INTERVALS FOR $\sigma=\frac{1}{2}$

Although Theorem 1 is valid for $\sigma=\frac{1}{2}$, one can do considerably better in this case. Namely, we have the following result of Balasubramanian-Ramachandra, which is stated here as

THEOREM 2. If $k \geqslant 1$ is a fixed integer, then for $C(\varepsilon, k) \log \log T \leqslant H \leqslant T / 2$ we have

$$
I_{1 / 2}(T+H, k)-I_{1 / 2}(T-H, k)=\int_{T-H}^{T+H}\left|\zeta\left(\frac{1}{2}+i t\right)\right|^{2 k} \mathrm{~d} t \geqslant\left(C_{k}^{\prime}-2 \varepsilon\right) H(\log H)^{k^{2}}
$$

where

$$
C_{k}^{\prime}=\frac{1}{\Gamma\left(k^{2}+1\right)} \prod_{p}\left\{\left(1-p^{-1}\right)^{k^{2}} \sum_{m=0}^{\infty}\left(\frac{\Gamma(k+m)}{\Gamma(k) m !}\right)^{2} p^{-m}\right\}
$$

The reader may find the proof e.g., in the comprehensive monograph of K. Ramachandra [31] (see in particular Remark 2 on p. 45). Therein a historical discussion is also given, as well as many related results involving $\zeta(s)$ and the more general Titchmarsh series, to which the methods of Balasubramanian-Ramachandra apply as well.

In what concerns upper bounds for $I_{1 / 2}(T+H, k)-I_{1 / 2}(T-H, k)$ there are no non-trivial results (apart from those coming from asymptotic formulas discussed before when $k=1$ or $k=2$ ). To obtain good bounds one then has to assume the Lindelöf hypothesis ( $\left.\mathrm{LH},\left|\zeta\left(\frac{1}{2}+i t\right)\right| \ll_{\varepsilon}|t|^{\varepsilon}\right)$ or the Riemann Hypothesis (RH) that all complex zeros of $\zeta(s)$ are on the "critical line" $\Re$ e $s=\frac{1}{2}$. Recall that both the $\mathrm{LH}$ and the $\mathrm{RH}$ are hitherto unproved, and that the $\mathrm{RH}$ implies the $\mathrm{LH}$, but whether the converse is true is not known. A classical result of J.E. Littlewood states (see e.g., [34] for a proof) that, under the $\mathrm{RH}\left(\log _{\ell} T=\log \left(\log _{\ell-1} T\right)\right)$,

$$
\left|\zeta\left(\frac{1}{2}+i t\right)\right| \ll \exp \left(C \frac{\log t}{\log _{2} t}\right) \quad(C>0),
$$

which gives a "trivial" upper bound for $I_{1 / 2}(T+H, k)-I_{1 / 2}(T-H, k)$. However, recently K. Soundararajan [33] complemented (7.1) in the case $H=T$ by obtaining, under the RH, the non-trivial upper bound

$$
\int_{0}^{T}\left|\zeta\left(\frac{1}{2}+i t\right)\right|^{2 k} \mathrm{~d} t \ll_{\varepsilon} T(\log T)^{k^{2}+\varepsilon}
$$

which is valid for any fixed $k>0$ and any given $\varepsilon>0$. In view of (7.1) this result, apart from ' $\varepsilon$ ', is therefore best possible. His method of proof is based on a large values estimate for $\log \left|\zeta\left(\frac{1}{2}+i t\right)\right|$, which gives as a corollary (under the $\mathrm{RH}$ ) the bound (7.3) with the explicit constant $C=3 / 8$. This improves the earlier value $C=0.4666 \ldots$ of Ramachandra-Sankaranarayanan [32].

Recently the author [19] used Soundararajan's method to obtain (under the RH) a bound for $I_{1 / 2}(T+H, k)-I_{1 / 2}(T-H, k)$. The result is contained in 
THEOREM 3. Let $H=T^{\theta}$ where $0<\theta \leqslant 1$ is a fixed number, and let $k$ be a fixed positive number. Then, under the $R H$, we have

$$
I_{1 / 2}(T+H, k)-I_{1 / 2}(T-H, k)=\int_{T-H}^{T+H}\left|\zeta\left(\frac{1}{2}+i t\right)\right|^{2 k} \mathrm{~d} t \ll H(\log T)^{k^{2}\left(1+O\left(1 / \log _{3} T\right)\right) .}
$$

Note that, since $H=T^{\theta}$ and $\theta$ is fixed, the right-hand side of (7.5) is

$$
\ll H(\log H)^{k^{2}\left(1+O\left(1 / \log _{3} T\right)\right)},
$$

which is more in tune with the lower bound in (7.1), although it does not seem possible to reach the range $\log _{2} T \ll H \leqslant T$ by the present method or to remove $O\left(1 / \log _{3} T\right)$ from the exponent in (7.5).

As already noted, upper bounds of the form

$$
I_{1 / 2}(T+H, k)-I_{1 / 2}(T-H, k) \ll H(\log T)^{k^{2}}
$$

can be derived unconditionally in the cases $k=1$ and $k=2$. They are known to hold for $\theta>1 / 3$ (and even for some slightly smaller values of $\theta$ ) when $k=1$, and for $\theta>2 / 3$ when $k=2$. In the case when $k=1 / 2$ it is known (see $\mathrm{K}$. Ramachandra [31]) that this bound holds unconditionally when $\theta>1 / 2$ and for $\theta>1 / 4$ under the RH. No other results of this type seem to be known for other values of $k$.

Theorem 3 will be deduced from a large values estimate for $\log \left|\zeta\left(\frac{1}{2}+i t\right)\right|$. This is

THEOREM 4. Let $H=T^{\theta}$ where $0<\theta \leqslant 1$ is a fixed number, and let $\mu(T, H, V)$ denote the measure of points $t$ from $[T-H, T+H]$ such that

$$
\log \left|\zeta\left(\frac{1}{2}+i t\right)\right| \geqslant V, \quad 10 \sqrt{\log _{2} T} \leqslant V \leqslant \frac{3 \log 2 T}{8 \log _{2}(2 T)} .
$$

Then, under the $R H$, for $10 \sqrt{\log _{2} T} \leqslant V \leqslant \log _{2} T$ we have

$$
\mu(T, H, V) \ll H \frac{V}{\sqrt{\log _{2} T}} \exp \left(-\frac{V^{2}}{\log _{2} T}\left(1-\frac{7}{2 \theta \log _{3} T}\right)\right),
$$

for $\log _{2} T \leqslant V \leqslant \frac{1}{2} \theta \log _{2} T \log _{3} T$ we have

$$
\mu(T, H, V) \ll H \exp \left(-\frac{V^{2}}{\log _{2} T}\left(1-\frac{7 V}{4 \theta \log _{2} T \log _{3} T}\right)^{2}\right),
$$

and for $\frac{1}{2} \theta \log _{2} T \log _{3} T \leqslant V \leqslant \frac{3 \log 2 T}{8 \log _{2}(2 T)}$ we have

$$
\mu(T, H, V) \ll H \exp \left(-\frac{1}{20} \theta V \log V\right) .
$$

To see how Theorem 4 implies Theorem 3 , first note that the contribution of $t$ satisfying $\log \left|\zeta\left(\frac{1}{2}+i t\right)\right| \leqslant \frac{1}{2} k \log _{2} T$ to the left-hand side of (7.5) is

$$
\leqslant H\left\{(\log T)^{k / 2}\right\}^{2 k}=H(\log T)^{k^{2}} .
$$


Likewise the bound (7.5) holds, by (7.7) and (7.8), for the contribution of $t$ satisfying $\log \left|\zeta\left(\frac{1}{2}+i t\right)\right| \geqslant 10 k \log _{2} T$. Thus we can consider only the range

$$
V+\frac{j-1}{\log _{3} T} \leqslant \log \left|\zeta\left(\frac{1}{2}+i t\right)\right| \leqslant V+\frac{j}{\log _{3} T}
$$

where $1 \leqslant j \ll \log _{3} T, V=2^{\ell-\frac{1}{2}} k \log _{3} T, 1 \leqslant \ell \leqslant \frac{3}{2}+\left[\frac{\log 10}{\log 2}\right]$. If we set

$$
U=U(V, j ; T):=V+\frac{j-1}{\log _{3} T}
$$

then we have

$$
\begin{aligned}
I_{1 / 2}(T+H, k)-I_{1 / 2}(T-H, k) & \ll H(\log T)^{k^{2}}+ \\
& +\log _{3} T \max _{U} \mu(T, H, U) \exp \left(2 k\left(U+\frac{1}{\log _{3} T}\right)\right),
\end{aligned}
$$

where $\mu(T, H, U)$ is the measure of $t \in[T-H, T+H]$ for which $\log \left|\zeta\left(\frac{1}{2}+i t\right)\right| \geqslant U$, and the maximum is over $U$ satisfying (7.12)-(7.13). If we use (7.7) and (7.8) of Theorem 2 , then in the relevant range for $U$ we obtain

$$
\begin{aligned}
\mu(T, H, U) \exp \left(2 k\left(U+1 / \log _{3} T\right)\right) & \ll H \log _{2} T \exp \left(2 k U-U^{2} G(T)\right), \\
G(T) & :=\frac{1}{\log _{2} T}\left(1+O\left(\frac{1}{\log _{3} T}\right)\right) .
\end{aligned}
$$

Since $\varphi(U)=2 k U-U^{2} G(T)$ attains its maximal value at $U=k / G(T)$, we have

$$
\begin{aligned}
& \mu(T, H, U) \exp \left(2 k\left(U+\frac{1}{\log _{3} T}\right)\right) \\
& \ll H \log _{2} T \exp \left(k^{2}\left(1+O\left(\frac{1}{\log _{3} T}\right)\right) \log _{2} T\right) \\
& =H(\log T)^{k^{2}\left(1+O\left(1 / \log _{3} T\right)\right)},
\end{aligned}
$$

so that (7.13) yields then (7.5) of Theorem 3.

The proof of Theorem 4 is based on the following lemmas, whose proofs may be found in [19] or [33].

LEMMA 1. Assume the RH. Let $T \leqslant t \leqslant 2 T, T \geqslant T_{0}, 2 \leqslant x \leqslant T^{2}$. If $\lambda_{0}=$ $0.4912 \ldots$ denotes the unique positive real number satisfying $\mathrm{e}^{-\lambda_{0}}=\lambda_{0}+\frac{1}{2} \lambda_{0}^{2}$, then for $\lambda \geqslant \lambda_{0}$ we have

$\log \left|\zeta\left(\frac{1}{2}+i t\right)\right| \leqslant \Re \mathrm{e}\left\{\sum_{2 \leqslant n \leqslant x} \frac{\Lambda(n)}{n^{\frac{1}{2}+\frac{\lambda}{\log x}+i t} \log n} \frac{\log (x / n)}{\log x}\right\}+\frac{(1+\lambda)}{2} \frac{\log T}{\log x}+O\left(\frac{1}{\log x}\right)$.

LEMMA 2. Assume the RH. If $T \leqslant t \leqslant 2 T, 2 \leqslant x \leqslant T^{2}, \sigma \geqslant \frac{1}{2}$, then

$$
\sum_{2 \leqslant n \leqslant x, n \neq p} \frac{\Lambda(n)}{n^{\sigma+i t} \log n} \frac{\log (x / n)}{\log x} \ll \log _{3} T .
$$


LEMMA 3. Let $2 \leqslant x \leqslant T, T \geqslant T_{0}$. Let $1 \ll H \leqslant T$ and $r \in \mathbb{N}$ satisfy $x^{r} \leqslant H$. For any complex numbers $a(p)$ ( $p$ denotes primes) we have

$$
\int_{T}^{T+H}\left|\sum_{p \leqslant x} \frac{a(p)}{p^{\frac{1}{2}+i t}}\right|^{2 r} \mathrm{~d} t \ll H r !\left(\sum_{p \leqslant x} \frac{|a(p)|^{2}}{p}\right)^{r} .
$$

Now we can give a sketch of the proof of Theorem 4. We assume the RH and let

$$
x=H^{A / V}, \quad z=x^{1 / \log _{2} T}, \quad A=A(T, V)(\geqslant 1),
$$

where $A$ will be suitably chosen below. We follow the method of proof of [33] (see also [19]) and accordingly consider three cases.

Case 1. When $10 \sqrt{\log _{2} T} \leqslant V \leqslant \log _{2} T$, we take $A=\frac{1}{2} \log _{3} T$.

Case 2. When $\log _{2} T \leqslant V \leqslant \frac{1}{2} \theta \log _{2} T \log _{3} T$, we take $A=\frac{\log _{2} T \log _{3} T}{2 V}$.

Case 3. When $\frac{1}{2} \theta \log _{2} T \log _{3} T \leqslant V \leqslant(3 \log 2 T) /\left(8 \log _{2} 2 T\right)$ we take $A=2 / \theta$.

Note that the last bound for $V$ comes from the bound (7.3) with $C=3 / 8$ (under the RH). Suppose that $\log \left|\zeta\left(\frac{1}{2}+i t\right)\right| \geqslant V \geqslant 10 \sqrt{\log _{2} T}$ holds. Then Lemma 1 and Lemma 2 yield

$$
V \leqslant S_{1}(t)+S_{2}(t)+\frac{1+\lambda_{0}}{2 A \theta} V+O\left(\log _{3} T\right)
$$

where we set

$$
S_{1}(t):=\left|\sum_{p \leqslant z} \frac{\log (x / p)}{\log x} p^{-\frac{1}{2}-\frac{\lambda_{0}}{\log x}-i t}\right|, S_{2}(t):=\left|\sum_{z<p \leqslant x} \frac{\log (x / p)}{\log x} p^{-\frac{1}{2}-\frac{\lambda_{0}}{\log x}-i t}\right| .
$$

This means that either

$$
S_{1}(t) \geqslant V_{1}=V\left(1-\frac{7}{8 A \theta}\right)
$$

or

$$
S_{2}(t) \geqslant \frac{V}{8 A \theta}
$$

since we easily get a contradiction if neither (7.16) nor (7.17) holds. Let now $\mu_{i}(T, H, V)(i=1,2)$ denote the measure of the set of points $t \in[T-H, T+H]$ for which (7.16) and (7.17) hold, respectively. Supposing that (7.16) holds then, by using Lemma 3 with $a(p)=\frac{\log (x / p)}{\log x} p^{-\lambda_{0} / \log x}$, we obtain

$$
\mu_{1}(T, H, V) V_{1}^{2 r} \leqslant \int_{T}^{T+H}\left|S_{1}(t)\right|^{2 r} \mathrm{~d} t \ll H r !\left(\sum_{p \leqslant z} \frac{1}{p}\right)^{r} .
$$

The condition in Lemma $3\left(x^{r} \leqslant H\right.$ with $\left.x=z\right)$ is equivalent to

$$
\frac{A r}{V \log _{2} T} \leqslant 1
$$


Recalling that

$$
\sum_{p \leqslant X} \frac{1}{p}=\log _{2} X+O(1),
$$

it follows that

$$
\log z=\frac{\log x}{\log _{2} T}=\frac{A \theta}{V \log _{2} T} \log T \leqslant \frac{\log T}{\log _{2} T},
$$

since $A \leqslant V$ in all cases. Therefore we have

$$
\sum_{p \leqslant z} \frac{1}{p} \leqslant \log _{2} T \quad\left(T \geqslant T_{0}\right) .
$$

Noting that Stirling's formula yields $r$ ! $\ll r^{r} \sqrt{r} \mathrm{e}^{-r}$, we infer from (7.18) and (7.20) that

$$
\mu_{1}(T, H, V) \ll H \sqrt{r}\left(\frac{r \log _{2} T}{\mathrm{e} V_{1}^{2}}\right)^{r} .
$$

In the Cases 1. and 2. and also in the Case 3. when $V \leqslant \frac{2}{\theta} \log _{2}^{2} T$, one chooses

$$
r=\left[\frac{V_{1}^{2}}{\log _{2} T}\right](\geqslant 1)
$$

With this choice of $r$ it is readily seen that (7.19) is satisfied, and (7.21) gives

$$
\mu_{1}(T, H, V) \ll H \frac{\sqrt{V}}{\log _{2} T} \exp \left(-\frac{V_{1}^{2}}{\log _{2} T}\right) .
$$

Finally in the Case 3 . when $\frac{2}{\theta} \log _{2}^{2} T \leqslant V \leqslant(3 \log 2 T) /\left(8 \log _{2} 2 T\right)$ and $A=2 / \theta$, we have

$$
V_{1}=V\left(1-\frac{7}{8 A \theta}\right)=V\left(1-\frac{7}{16}\right)>\frac{V}{2} .
$$

Thus with the choice $r=[V / 2]$ we see that (7.19) is again satisfied and

$$
\sqrt{r}\left(\frac{r \log _{2} T}{\mathrm{e} V_{1}^{2}}\right)^{r} \leqslant \sqrt{V}\left(\frac{2 \log _{2} T}{\mathrm{e} V}\right)^{r} \leqslant V^{\frac{1}{2}-\frac{r}{4}} \ll \exp \left(-\frac{1}{10} V \log V\right)
$$

giving in this case

$$
\mu_{1}(T, H, V) \ll H \exp \left(-\frac{1}{10} V \log V\right) .
$$

We bound $\mu_{2}(T, H, V)$ in a similar way by using (7.17). It follows, again by Lemma 3, that

$$
\begin{aligned}
\left(\frac{V}{8 A \theta}\right)^{2 r} \mu_{2}(T, H, V) & \leqslant \int_{T}^{T+H}\left|S_{2}(t)\right|^{2 r} \mathrm{~d} t \\
& \ll H r !\left(\sum_{z<p \leqslant x} \frac{1}{p}\right)^{r}=H r !\left(\log _{2} x-\log _{2} z+O(1)\right)^{r} \\
& \ll H\left\{r\left(\log _{3} T+O(1)\right)\right\}^{r}
\end{aligned}
$$


We obtain

$$
\mu_{2}(T, H, V) \ll H\left(\frac{8 A}{V}\right)^{2 r}\left(2 r \log _{3} T\right)^{r} \ll H \exp \left(-\frac{V}{2 A} \log V\right) .
$$

Namely the second inequality in (7.24) is equivalent to

$$
\left(\frac{A}{V}\right)^{2} r \log _{3} T \ll \exp \left(-\frac{V}{2 r A} \log V\right) .
$$

In all the Cases 1.-3. we take

$$
r=\left[\frac{V}{A}-1\right](\geqslant 1) .
$$

The condition $x^{r} \leqslant H$ in Lemma 3 is equivalent to $r A \leqslant V$, which is trivial with the above choice of $r$. To establish (7.25) note first that

$$
\left(\frac{A}{V}\right)^{2} r \log _{3} T \leqslant \frac{A}{V} \log _{3} T .
$$

In the Case 1 . the second expression in (7.26) equals $\log _{3}^{2} T /(2 V)$, while

$$
\exp \left(-\frac{V}{2 r A} \log V\right)=\exp \left(-\left(\frac{1}{2}+o(1)\right) \log V\right)=V^{-1 / 2+o(1)}
$$

Therefore it suffices to have

$$
\frac{\log _{3}^{2} T}{V} \ll V^{-1 / 2+o(1)}
$$

which is true since $10 \sqrt{\log _{2} T} \leqslant V$. In the Case 2 . the analysis is similar. In the Case 3 . we have $A=2 / \theta$, hence $\left(A \log _{3} T\right) / V \ll\left(\log _{3} T\right) / V$ and

$$
\exp \left(-\frac{V}{2 r A} \log V\right)=\exp \left(-\left(\frac{1}{2} \theta+o(1)\right) \log V\right)=V^{-\left(\frac{1}{2} \theta+o(1)\right)},
$$

so that (7.25) follows again. Thus we have shown that in all cases

$$
\mu_{2}(T, H, V) \ll H \exp \left(-\frac{V}{2 A} \log V\right) .
$$

Theorem 2 follows now from (7.21), (7.22) and (7.27). Namely in the Case 1. we have

$$
\frac{V_{1}^{2}}{\log _{2} T}=\frac{V^{2}\left(1-\frac{7}{4 \theta \log _{3} T}\right)^{2}}{\log _{2} T} \leqslant V \frac{\log V}{\log _{3} T}=\frac{V \log V}{2 A},
$$

which gives (7.7). If the Case 2. holds we have again

$$
\frac{V_{1}^{2}}{\log _{2} T}=\frac{V^{2}\left(1-\frac{7 V}{4 \theta \log _{2} T \log _{3} T}\right)^{2}}{\log _{2} T} \leqslant \frac{V^{2} \log V}{\log _{2} T \log _{3} T}=\frac{V \log V}{2 A},
$$


and (7.8) follows. In the Case 3. when $\frac{1}{2} \theta \log _{2} T \log _{3} T \leqslant V \leqslant \frac{2}{\theta} \log _{2}^{2} T$ we have

$$
\begin{aligned}
\mu(T, H, V) & \ll H \exp \left(-\frac{V_{1}^{2}}{\log _{2} T}\right)+H \exp (-\theta V \log V) \\
& \ll H \exp \left(-\frac{\theta}{20} V \log V\right),
\end{aligned}
$$

since

$$
\frac{V_{1}^{2}}{\log _{2} T} \geqslant \frac{V^{2}}{4 \log _{2} T} \geqslant \frac{\theta V \log _{2} T \log _{3} T}{8 \log _{2} T} \geqslant \frac{\theta}{20} V \log V .
$$

In the remaining range of Case 3 . we have

$$
\begin{aligned}
\mu(T, H, V) & \ll H \exp \left(-\frac{1}{10} V \log V\right)+H \exp \left(-\frac{V}{2 A} \log V\right) \\
& \ll H \exp \left(-\frac{1}{10} V \log V\right)+\exp \left(-\frac{\theta}{4} V \log V\right),
\end{aligned}
$$

and (7.9) follows. The proof of Theorem 4 is complete. 


\section{REFERENCES}

[1] F.V. Atkinson, The mean value of the Riemann zeta-function, Acta Math. 81(1949), 353-376.

[2] R. Balasubramanian, An improvement on a theorem of Titchmarsh on the mean square of $\left|\zeta\left(\frac{1}{2}+i t\right)\right|$, Proc. London Math. Soc. (3) 36(1978), 540-576.

[3] R. Balasubramanian, A. Ivić and K. Ramachandra, The mean square of the Riemann zetafunction on the line $\sigma=1$, L'Enseignement Math. 38(1992), 13-25.

[4] R. Balasubramanian, A. Ivić and K. Ramachandra, An application of the Hooley-Huxley contour, Acta Arith. 65(1993), 45-51.

[5] J.B. Conrey, D.W. Farmer, J.P. Keating, M.O. Rubinstein and N.C. Snaith, Integral moments of $L$-functions, Proc. Lond. Math. Soc., III. Ser. 91(2005), 33-104.

[4] J.L. Hafner and A. Ivić, On the mean square of the Riemann zeta-function on the critical line, J. Number Theory 32(1989), 151-191.

[7] D.R. Heath-Brown, The twelfth power moment of the Riemann zeta-function, Quart. J. Math. Oxford 29(1978), 443-462.

[8] D.R. Heath-Brown, The fourth moment of the Riemann zeta-function, Proc. London Math. Soc. (3)38(1979), 385-422.

[9] M.N. Huxley and A. Ivić, Subconvexity for the Riemann zeta-function and the divisor problem, Bulletin CXXXIV de l'Académie Serbe des Sciences et des Arts - 2007, Classe des Sciences mathématiques et naturelles, Sciences mathématiques No. 32, pp. 13-32.

[10] A.E. Ingham, Mean-value theorems in the theory of the Riemann zeta-function, Proc. London Math. Soc. (2)27(1926), 273-300.

[11] A. Ivić, The Riemann zeta-function, John Wiley \& Sons, New York 1985 (2nd edition. Dover, Mineola, New York, 2003).

[12] A. Ivić, The mean values of the Riemann zeta-function, LNs 82, Tata Inst. of Fundamental Research, Bombay 1991 (distr. by Springer Verlag, Berlin etc.).

[13] A. Ivić, On the fourth moment of the Riemann zeta-function, Publs. Inst. Math. (Belgrade) 57(71)(1995), 101-110.

[14] A. Ivić, Some problems on mean values of the Riemann zeta-function, J. de Théorie des Nombres de Bordeaux 8(1996), 101-123.

[15] A. Ivić, On the ternary additive divisor problem and the sixth moment of the zeta-function, "Sieve Methods, Exponential Sums, and their Applications in Number Theory" (eds. G.R.H. Greaves, G. Harman, M.N. Huxley), Cambridge Univ. Press, Cambridge, 1996, 205-243.

[16] A. Ivić, On the error term for the fourth moment of the Riemann zeta-function, J. London Math. Soc. 60(2)(1999), 21-32.

[17] A. Ivić, On the integral of the error term in the fourth moment of the Riemann zeta-function, Functiones et Approximatio, 28(2000), 37-48.

[18] A. Ivić, On mean values of some zeta-functions in the critical strip, Journal de Théorie des Nombres de Bordeaux 15(2003), 163-178.

[19] A. Ivić, Moments of the zeta-function in short intervals, to appear, see arXiv:0807.1181.

[20] A. Ivić and K. Matsumoto, On the error term in the mean square formula for the Riemann zeta-function in the critical strip, Monatshefte Math. 121(1996), 213-229.

[21] A. Ivić and Y. Motohashi, The mean square of the error term for the fourth moment of the zeta-function, Proc. London Math. Soc. (3)66(1994), 309-329.

[22] A. Ivić and Y. Motohashi, The fourth moment of the Riemann zeta-function, J. Number Theory 51(1995), 16-45.

[23] A. Ivić and Y. Motohashi, The moments of the Riemann zeta-function Part I: The fourth moment off the critical line, Functiones et Approximatio 35(2006), 133-181.

[24] A. Ivić, M. Jutila and Y. Motohashi, The Mellin transform of powers of the zeta-function, Acta Arith. 95(2000), 305-342.

[25] K. Matsumoto, The mean square of the Riemann zeta-function in the critical strip, Japanese J. Math. 15(1989), 1-13.

[26] K. Matsumoto, Recent developments in the mean square theory of the Riemann zeta and allied functions, in "Number Theory", eds. R.P. Bambah et al., Hindustan Book Agency \& Indian National Science Academy, Birkhäuser, 2000, pp. 241-286. 
[27] K. Matsumoto and T. Meurman, The mean square of the Riemann zeta-function in the critical strip II, Acta Arith. 68(1994), 369-382, and III ibid. 64(1993), 357-382.

[28] Y. Motohashi, An explicit formula for the fourth power mean of the Riemann zeta-function, Acta Math. 170(1993), 181-220.

[29] Y. Motohashi, Spectral theory of the Riemann zeta-function, Cambridge University Press, Cambridge, 1997.

[30] K. Ramachandra, Some problems of analytic number theory I, Acta Arith. 31(1976), 313-324.

[31] K. Ramachandra, On the mean-value and omega-theorems for the Riemann zeta-function LNs 85, Tata Inst. Fundamental Research, Bombay, 1995 (distr. by Springer Verlag, Berlin etc.).

[32] K. Ramachandra, A. Sankaranarayanan, On some theorems of Littlewood and Selberg. I. J. Number Theory 44(1993), 281-291.

[33] K. Soundararajan, Moments of the Riemann zeta-function, Annals of Math. (2008), to appear, see arXiv:0806.0786.

[34] E.C. Titchmarsh, The theory of the Riemann zeta-function (2nd edition), University Press, Oxford, 1986.

Aleksandar Ivić, Katedra Matematike RGF-a Universiteta u Beogradu, Đušina 7, 11000 BeOgrad, Serbia.

E-mail address: aivic_2000@yahoo.com, ivic@rgf.bg.ac.yu

Received on March 7, 2009

Accepted on March 30, 2009. 